\title{
DIGITALCOMMONS
}

—@WAYNESTATE -

Wayne State University

$1-1-2014$

\section{Go to the Hospital or Stay at Home? A Qualitative Study of Expected Hospital Decision Making among Older African Americans with Advanced Heart Failure}

Faith Pratt Hopp

Wayne State University, bb2938@wayne.edu

Christina Marsack

Wayne State University, christina.marsack@gmail.com

Jessica Camp

Wayne State University, jkcamp81@gmail.com

Shirley Ann Thomas

Wayne State University, sathomas@wayne.edu

\section{Recommended Citation}

Hopp, F. P., Marsack, C., Camp, J. K., \& Thomas, S. (2014). Go to the Hospital or Stay at Home? A Qualitative Study of Expected Hospital Decision Making among Older African Americans with Advanced Heart Failure. Journal of Gerontological Social Work, 57(1), 4-23. doi: 10.1080/01634372.2013.848966.

Available at: http://digitalcommons.wayne.edu/soc_work_pubs/17

This Article is brought to you for free and open access by the Social Work at DigitalCommons@WayneState. It has been accepted for inclusion in Social Work Faculty Publications by an authorized administrator of DigitalCommons@WayneState. 
NOTICE IN COMPLIANCE WITH PUBLISHER POLICY: This is an Author's Accepted Manuscript of an article published in Journal of Gerontological Social Work 57(1), 2014, available online: http://www.tandfonline.com/10.1080/01634372.2013.848966. It has been formatted for archiving; pagination has been added for this version. 


\title{
Go to the Hospital or Stay at Home? A Qualitative Study of Expected Hospital Decision Making among Older African Americans with Advanced Heart Failure
}

\author{
F. P. Hopp, Associate Professor \\ C. Marsack, Graduate Student \\ J. K. Camp, Graduate Student \\ S. Thomas, Clinical Assistant Professor \\ Wayne State University School of Social Work
}

Corresponding Author: Faith Hopp, 136 Thompson Home, 4756 Cass

Ave., Detroit, Ml 48202 | (313) 5774423 | Fax (313) 5778770 |

faithhopp@wayne.edu

\begin{abstract}
To address the need for more information concerning hospital decision making, we conducted in-depth interviews among African Americans with heart failure and their family caregivers ( $n=11$ dyads). Using a case scenario, we asked participants about their anticipated hospitalization decisions. Most patients indicated that they would seek care to avoid further deterioration or death from their worsening condition. Many family caregivers anticipated having an active influence on hospitalization decisions. Findings suggest that social workers should encourage the development of adequate home-based services, recognize diverse communication styles, and use this information to facilitate medical decision making by these patients and their caregivers.
\end{abstract}

Keywords: Heart failure, Hospital, Decision-making, African American

Although heart failure is the most common reason for hospitalization among older adults (Freidman, Jiang, \& Russo, 2009; Hedrick, 2011), very little in-depth information is available concerning the way in which persons with heart failure make hospitalization decisions. An estimated 5.7 million adults in the United States are currently coping with heart failure. African Americans are especially vulnerable to this condition and 


\section{F. P. Hopp et al.}

are three to seven times more likely to have contributing medical issues, such as hypertension (Yancy, 2005), and 50\% more likely to have heart failure (Franciossa, Ferdinand, \& Yancy, 2010). Moreover, some studies have found that African Americans have higher rates of heart-failurerelated hospitalizations compared with Caucasians (Deswal, Petersen, Urbauer, Wright, \& Beyth, 2006; Husaini et al., 2011). Historically African Americans have experienced inequality in the quality of health care they have received, and consequently may distrust the medical system, preferring active treatment over palliative care (Allen, Allen, Hilgeman, \& DeCoster, 2008; Braun, Beyth, Ford, \& McCullough, 2008; Givens Tjia, Zhou, Emanuel, \& Ash, 2010; LaVeist, Nickerson \& Bowie, 2000). These factors can have important implications for how African Americans approach medical care, their access to services, and their desire for medical information and treatment (Torke, Corbie-Smith, \& Branch, 2004).

The rapid increase in heart failure in the United States, growing use of hospital services and associated costs, along with the burgeoning number of African Americans with heart failure, are factors suggesting the need for a more in-depth examination of hospitalization decision making among this population (Husaini et al., 2011). An extensive review of the existing literature found only one study (Fried et al., 2000) examining hospital decision making among African Americans. Addressing this knowledge gap is a critical first step in addressing the need for supportive, community-based services for this population. Hospitalization has usually been understood to mean inpatient care (Husaini et al., 2011). However, decision making for hospitalization has not been explicitly defined in the literature. For this study, we define hospital decision making as the initial decision to seek care at an inpatient medical facility when faced with symptoms related to serious illness, regardless of whether the attempt to seek hospital care results in an actual inpatient stay.

Geriatric social workers provide extensive community-based services to persons with chronic illness, such as heart failure. They can play a key role in providing psychosocial care and support, helping people make important choices about home versus hospital care amidst the often changing and uncertain trajectory of advanced illness (Hebert, Schulz, Copeland, \& Arnold, 2009; Hobart, 2002). However, more information on expectations concerning hospital decision making is critically needed to help social workers and other health care professionals gain a greater understanding of the decision-making process, develop culturally 
Decisions in Heart Failure

sensitive interventions, and refine strategies for addressing conflicts between care recipients and their caregivers.

This qualitative study explores the perceptions of African Americans with heart failure, and their caregivers, concerning hospitalization. Specifically, we conducted interviews to obtain information about the expectations that African Americans with heart failure may have during times they are having symptoms and may think about whether to go to the hospital or instead manage their symptoms at home. We conducted separate interviews with persons with heart failure and their caregivers. We defined caregiver dyads as consisting of a person with heart failure and that person's primary caregiver. The caregivers in this study were those nominated by the person with heart failure to be invited to participate in the study and who consented to study participation. They included adult children, adult grandchildren, one neighbor, and one spouse. All of the persons with heart failure and their caregivers selfidentified as African American. The specific research questions addressed were: 1) When faced with a deteriorating medical condition, what are the expectations of older African Americans with heart failure and their caregivers concerning going to the hospital or staying at home? 2) What are the reasons provided by older African Americans with heart failure for going to the hospital vs. staying at home? 3) What communication styles are evident between African Americans with heart failure and their caregivers in their discussions of future hospital decision making?

\section{Literature Review}

Decisions about hospitalization often take place within a broader context of advanced illness, including the choice between accessing home or hospital care. The primary focus of existing literature has been on the role of health care providers, particularly physicians, in decisions about health care and treatment. For example, researchers have noted the "prognostic uncertainty and complex trade-offs in the choice of medical care" associated with heart failure decisions (Allen et al., 2012). However, there has been greater emphasis in recent years on the importance of communication between health care providers and family members (Shin et al., 2013). For example, the American Heart Association notes that, when medical professionals engage in open, clear, and accurate communication with heart-failure patients and their family members, health-care decisions are better aligned with their overall treatment goals 


\section{F. P. Hopp et al.}

(Allen et al., 2012). Because the caregivers of older adults often play a key role in health care decisions, such communication is essential (Lyons et al., 2006), particularly when such decisions that take place at the end of life (Haley, Allen, Reynolds, Chen, Burton, \& Gallagher-Thompson, 2002). Greater exploration of this medical decision-making process, particularly as it relates to hospitalization decisions, is a crucial part of understanding how patients make medically informed decisions. It also affects the psychological outcomes for family caregivers coping with the uncertain and unpredictable heart failure trajectory.

Research on interpersonal communication within health care environments has highlighted the challenges and opportunities related to patient-caregiver communication in health care settings. The concept of 'shared care' refers to communication styles in close relationships that emphasize reciprocity and sharing in decision making about healthrelated issues, and the extent of shared care has been associated with greater confidence in self-care activities (Sebern \& Riegel, 2009). However, the degree of harmony on medical decisions has varied (Horowitz, Goodman, \& Reinhardt, 2004; Lyons, Zarit, Sayer, \& Whitlach, 2002; Sebern \& Reigel, 2009). A recent qualitative interview study, which included 17 heart failure patient and caregiver dyads, found that persons with heart failure and their caregivers do not always agree about heartfailure management or appropriate ways to handle conflicting emotions. They may also have different perspectives about future care and treatment (Retrum, Nowels, and Bekelman, 2012). The existing research also suggests that there are a variety of approaches to health-care decision making, including those focused on autonomy of the elder, as well as those involving collaboration between elders and caregivers (Nakashima, Chapin, Macmillan, \& Zimmerman, 2005). Clinicians and researchers have recently emphasized the importance of shared decision-making and patient-centered care as essential components of ethical and humane care for persons with serious illness. Munthe, Sandman, and Cutas (2012) have identified the key elements of the communication components associated with patient-centered care and shared decision making that are essential for ethical patient care. These elements include 1) attention to the importance of the patient narrative, which includes life stories and interpretations by the person with a serious illness in light of life experiences; 2) collaboration, in which both the person with serious illness and their caregivers share in making important decisions about care and treatment; and 3) continuity, described as an ongoing process of decision- 
Decisions in Heart Failure

making that takes into account changes in the patient narrative and ongoing revision of the shared decision-making process. Thorne, Oliffe, and Stadjuhar (2013), in their qualitative analysis of 60 persons undergoing cancer treatment, note the unique challenges of such an approach in the health care environment. These challenges include a communicative environment that is characterized by diversity in cultural experiences and expectations around illness, changing technologies, the complexity of medical treatments, and the stresses and strains associated with making complex decisions in health care settings that are often confusing and impersonal. They note that within such a communication context, communication 'breakdowns' can occur between persons with serious illness and their caregivers. Given these challenges, there is a critical need to build on this knowledge base with qualitative, in-depth research that can guide social workers and other health-care professionals in providing appropriate treatment and decision-related support.

\section{Racial Disparities in Heart Failure}

Understanding the decision-making process among African Americans with heart failure is critical, as this racial group has been found to experience health disparities both in the prevalence of the disease and its treatment. In general, African Americans are much more likely than Caucasians to experience heart failure, and they experience heart failure at much younger ages (Mitchell et al., 2011). The American Heart Association (2013) states that, among non-Hispanic African Americans over the age of twenty, nearly $6.8 \%$ of men and $7.1 \%$ of women have chronic heart disease. In addition to this, African Americans and Hispanics over the age of 65 who have Medicare are more likely to be hospitalized with heart failure and are more likely to be discharged to their home rather than to a long-term care facility, compared to Caucasians (Brown, Haldeman, Croft, Giles, \& Mensah, 2005). Being discharged home without any type of follow up or support care can lead to re-hospitalization, which concerns practitioners and researchers, as readmission rates are significantly higher for African Americans dealing with heart failure and may be an underlying factor in examining the root of racial disparities in heart-failure treatment (Joynt, Orav, \& Jha, 2011).

\section{Decision making and African Americans}

Health care disparities have had a profound influence on African 


\section{F. P. Hopp et al.}

Americans' attitudes towards health care and medical decisions. For example, a qualitative, focus-group study among persons who had received treatment for ischemic heart disease in two urban, university hospitals (Ferguson et al., 1998) found that African Americans were particularly likely to mention perceived discrimination, perceptions of the patient-physician relationship, and spiritual issues as factors that influenced decision making regarding cardiac care. A second study of African-American cancer patients, found that mistrust of the medical community, concerns about privacy, and perceived stigma concerning seeking help for emotional support were cited as barriers in seeking treatment for cancer among African Americans (Matthews, Sellergren, Manfredi, \& Williams, 2010). While these studies give important insight into medical decision making among African Americans, very little information is available concerning hospital decision making from the perspective of these patients and their caregivers (Torke, Corbie-Smith, \& Branch, 2004). Moreover, few studies (e.g., Horowitz, Rein, and Leventhal, 2004; Zambroski, 2003), have explored the perceptions of African Americans with regards to health care decision making, and the ethnicity or race of study participants is often not identified. The higher incidence of heart failure and lower survival rates for that condition among African Americans (Loehr, Rosamond, Chang, Folsom \& Chambless, 2008), suggests the need to address diversity with respect to decision making for persons with for heart failure (Allen, Arslanian-Engoren, \& Lynch-Sauer, 2008).

\section{Methods}

This study is a secondary analysis of a qualitative dataset that used separate interviews with heart-failure patients and their caregivers as a means of seeking in-depth information on perceptions and attitudes concerning medical decision making.

\section{Sample}

The secondary data utilized for the present study were obtained from an Institutional Review Board-approved study designed to explore the lived experience of advanced heart failure among urban, African-American elders (Hopp, Thornton, Martin, \& Zalenski, 2011). The purpose of this larger study was to understand the experiences of persons with heart failure and their caregivers concerning opportunities and barriers to heart- 


\section{Decisions in Heart Failure}

failure care. The study context was an urban teaching hospital located in the Midwestern region of the United States. We recruited African Americans, aged 60 and older, with advanced heart failure, as defined by established clinical algorithms (Dolgin, 1994; Lee et al., 2003). In order to qualify for the study, prospective participants were required to have a primary diagnosis of heart failure, as identified in the hospital discharge record, and to meet other initial criteria for inclusion: a diagnosis of advanced heart failure, defined as a one-year mortality risk of $32.5 \%$ or above, according to the risk assessment tool developed by Lee and colleagues (Lee et al., 2003). In order to confirm that heart failure was advanced, we also required that prospective participants have had three or more hospitalizations with a diagnosis of heart failure in the three years prior to hospital record review. They also had to have the cognitive ability to consent to the study and to participate in focus-groups or interviews. We determined this by reviewing hospital records and excluding those who had dementia-related diagnoses. We limited study participation to those who self-identified as African Americans, since they were the focus of the study and because they comprised the largest racial group of patients at the study site. To respect their need for privacy and recuperation, we waited to contact potential participants until they had been home from the hospital for at least two weeks.

Potential participants were sent a letter about the study and were also contacted by telephone. The letter informed them that the researchers were interested in learning more about their heart failure experience. Of the 115 prospective participants who were contacted, 62 refused to participate, three died prior to data collection, 14 did not complete the appointment process or where no-shows, and one consented to the study but technical issues prevented an available recorded transcript. A total of 35 persons agreed to participate and had completed interview or focus group records, representing a participation/completion rate of $30 \%$ (35/115). Of these, 13 persons participated in focus groups and 22 participated in individual interviews.

We recruited caregivers using a two-step process in which participants with heart failure consented to participate in the study, and were then asked if they would give written permission for the research assistant to contact a person that they named as a caregiver (family member or friend) who could be contacted and invited to be interviewed. There were no requirements or restrictions placed on the type of person 


\section{F. P. Hopp et al.}

who could be nominated or on the type and quantity of care required for a person to be defined as a caregiver for a person with heart failure. Of the 22 persons with individual interviews from the parent study, 12 did not have a caregiving to nominate or did not wish to nominate their caregiver, and 10 nominated a caregiver who also agreed to be interviewed.

The present study is a secondary analysis of patient/caregiver dyads. A dyad is defined as a person with heart failure and that person's caregiver. For this study, we created the dyad pairs by combining qualitative data obtained from individual interviews conducted among people with heart failure with qualitative data obtained from separate interviews with the caregivers of these individuals. A total of ten older adults nominated a single caregiver who subsequently agreed to participate in the study. One older adult identified two caregivers, both of whom agreed to participate, resulting in a total of 11 dyads.

\section{Data Collection}

Participants completed a questionnaire that included questions about their heart-related health status, using the Kansas City Cardiomyopathy Questionnaire (KCCQ, Cardiovascular Outcomes, 2009). Caregivers completed a questionnaire covering basic demographic information and self-reported health status.

As part of a semi-structured interview, persons with heart failure were asked to consider this scenario:

Imagine that you have had a large heart attack and your heart function is very weak. For the past year, you've been increasingly unable to lead an independent life because of fatigue. Your heart function is so poor that you're short of breath with simple activities, and your legs are usually swollen, even when you're sitting down. The treatments you're receiving are becoming less and less effective. Today, your symptoms are as bad as they've ever been, and your doctor wants you to come to the hospital for more of the same treatments. So your option is to either go to the hospital for treatment or to stay at home. What would you do?

Caregivers were given a similar scenario in which they were asked about their perceptions of the views of their loved one concerning hospitalization, versus staying at home. For example, the last sentence for 


\section{Decisions in Heart Failure}

the caregiver interview was phrased as "What do you think [he/she] would do in this situation?" Interviewers used probes to elucidate the reasons given by persons with heart failure and their caregivers for their expected choice.

\section{Analysis procedure}

The first author had primary responsibility for analysis of the data, and met regularly with a writing group to develop the themes and refine the findings from the analysis. To examine responses to the first research questions concerning preferences for going to the hospital versus staying at home, the author reviewed each pair of interviews to classify the expected choice identified by the patient and caregiver. This information was utilized to derive the presence of agreement or disagreement within each dyad. To evaluate responses to the second research question concerning reasons for hospitalization, answers were reviewed from all participants (patients and caregivers) to determine the reasons for going to the hospital versus staying at home. The author followed a thematic analysis process (Braun \& Clarke, 2006). First, the taped interviews and transcripts were reviewed to gain familiarity with the data. The second phase involved generation of initial codes. In the third phase, broader themes were created based on the interviews, through a process of sorting and combining codes to fit within appropriate theme categories. In the fourth phase, the author carefully reviewed these themes and refined them by re-reading the quotes and ensuring that they fit within the thematic structure. The entire set of data was then reviewed to ensure congruence between codes and themes. In the fifth and final stage, quotes were selected that provided accurate and vivid descriptions of the overarching themes.

For the third research question, which examined the ways patients and caregivers anticipated they would discuss these important health decisions, a side-by-side comparison was made of the qualitative responses to the illness scenario for each dyad. An inductive process was utilized to derive communication styles from the data in two steps. First, data from all of the dyads were reviewed to derive a coding scheme for each dyad's communication style. The data was then reviewed to ensure that the five styles that we identified comprehensively represented all observed communication patterns.

The analytic plan included two steps to ensure rigor (Padgett, 1998). 


\section{F. P. Hopp et al.}

First, the first author reviewed all of the study findings, associated quotes, and study conclusions with the last author, who was not involved in the initial data collection but had extensive social work practice experience with diverse groups of older adults. Second, none of the study authors or the research assistant was employed by the hospital study site, assuring we could bring a more objective perspective to our observations and analysis.

\section{Findings}

\section{Patient and Caregiver Characteristics}

Characteristics of patients and caregivers are shown in Table 1. Overall means for participants on the Kansas City Cardiomyopathy (KCCQ) scale, scored from 1 to 100, with higher scores indicated a higher level of functioning, ranged from 16.67 to 83.33 for physical limitations and from 0.00 to 100.00 for social limitations. Patient education ranged from six to 16 years. Five of the persons with heart failure resided separately from their caregivers and five resided with their caregivers. The most common relationship between caregivers and patients was that of adult child $(n=7)$; one caregiver was a neighbor, two were adult grandchildren, and one was a spouse. Most of the caregivers reported their health status as good $(n=6)$ or very good $(n=3)$, with smaller numbers reporting their health as poor $(n=1)$ or excellent $(n=1)$.

\section{Research Question \#1: Expectations of going to the hospital versus staying at home.}

As shown in Table 1, the majority of those with heart failure (nine out of ten) indicated that they would choose to go to the hospital in reacting to the illness scenario. In seven of the eleven dyads, both the patient and caregiver said that the person with heart failure would want to go to the hospital. In one of the dyads (dyad \#11) both the patient and the caregiver agreed that the patient would want to stay at home. Two dyads disagreed. The patient in dyad \#2 indicated that she would want to go to the hospital, but her caregiver expected that she would want to stay at home. In dyad \#3, the individual with heart failure indicated that she would want to stay at home, while the caregiver thought she would want to go to the hospital. 
Decisions in Heart Failure

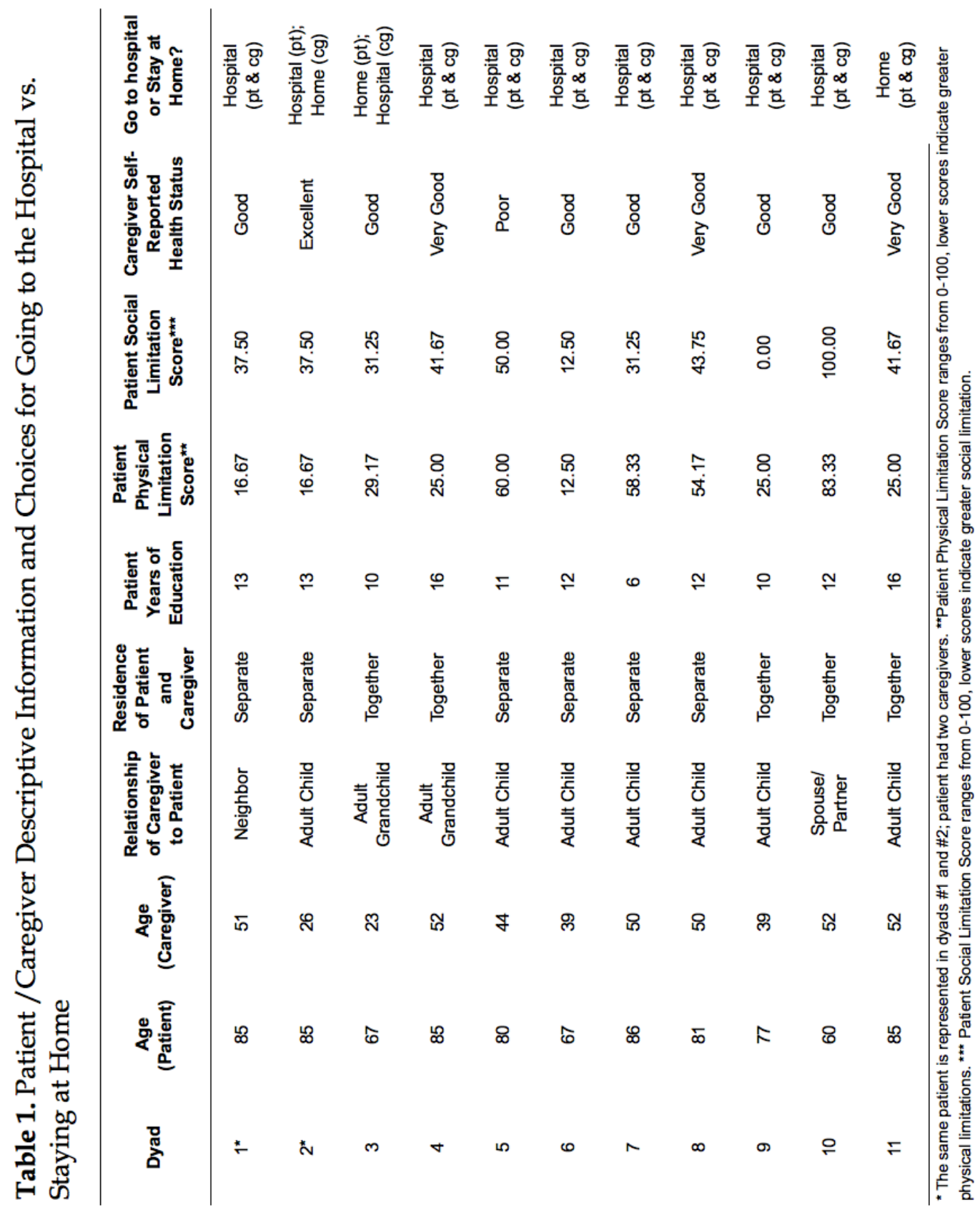




\section{F. P. Hopp et al.}

\section{Research Question \#2: Reasons for going to the hospital versus staying at home.}

Findings of the second research question revealed five key themes: selfmonitoring, avoiding death, relying on medical authorities, challenges of hospital care, and the availability of resources at home. These themes are summarized below.

Self-monitoring. We defined "self-monitoring" as keeping track of symptoms and going to the hospital to ascertain their meaning and significance. This theme was evident in three of the dyads. For example, the son of an 87-year-old woman with heart failure noted that, "she would want to see what was going on, what was wrong with her and why she was feeling this way and why she's so weak in the legs..." A neighbor of an 85-year-old woman with heart failure noted that he would take her to the hospital because he did not think her blood pressure cuff was giving an accurate reading.

Avoiding death. The second theme, going to the hospital as a means of avoiding death at home, was identified by four of the dyads. For example, the neighbor of the 85-year-old woman mentioned above pointed out that, had she been alone at night, "She probably would have died that night", while a 77-year-old woman with heart failure indicated a preference for hospital care, stating "You gonna die anyway, if you stay at home. At least you've got a chance when you go in." These statements acknowledge the possibility of death, and the hope that hospital care presents a possible way of avoiding it.

Relying on medical authorities. A third theme, which was identified by three of the dyads, is the perceived need to rely on medical authorities. An 86-year-old woman with heart failure noted that "on my regular visits to him, if he discovers I need to go over to the hospital, I'm gone...he gets mad each time I walk in 'Oh! You back here again!' I want to slap their face. 'Don't you know if I wasn't sick I wouldn't be here [laughs]?"The grandson of a 77-year-old woman with heart failure mentioned educational qualifications, noting that "They can do it better than I can. They've got the PhDs, not me."

Challenges of hospital care. Despite preferences for hospitalization, the challenges of hospital care emerged as a theme for three of the dyads. For example, the grandson of the 85-year-old woman with heart failure mentioned that hospitalization for his grandmother involves "Being 
Decisions in Heart Failure

probed and stuck with needles and therapy..."; his observation that " I know what she goes through..." and his view that "She don't like it, but you've got to be doing it". A 77- year-old man with heart failure noted other challenges, "being tied down" and "the crazy nurse". These responses indicate that many respondents anticipate that their decisions to go to the hospital will be accompanied by challenges, including discomfort and pain.

Availability of resources at home. A fifth theme, which was evident for five of the dyads, related to reasons for going to the hospital concerned perceptions around the availability of resources at home, or lack thereof. For example, one caregiver of a 67-year-old woman explained that she would urge her loved one to go to the hospital "because staying at home, it wouldn't help her. She has nothing here at the house...". In contrast, another 67-year-old woman expressed her view that resources were sufficient for staying at home, "if [home care service providers] come like they been coming, it would be about the same [as the hospital]."

\section{Research Question \#3: Dyad communication styles.}

Thematic analysis revealed five distinct communication styles: influential, complementary-influential, complementary, conditional, and directive.

Influential communication style. The communication style for one of the dyads can be described as an influential communication style. The influential communication style is one in which the person with heart failure and their caregiver have different expected preferences for going to the hospital vs. staying at home, and the caregiver perceives the need to persuade their loved one to go to follow the caregiver's preferences. For example, a person with heart failure noted that "I'd stay at home... I wouldn't take a risk because I'd wonder what they'd be doing here at the house... I'd rather stay home and get my treatments.". The caregiver, in contrast, notes that she would "advise" their loved one to go to the hospital, noting that this advice would be given because "...she wouldn't want to be sick no more."

Complimentary-influential communication style. A complimentaryinfluential style, which was present in five of the dyad pairs, is one in which both the person with heart failure and their caregiver have similar views about hospitalization, but the caregiver nonetheless feels the need to persuade their loved one to make this decision. For example, the patient in one dyad pair expressed that they would go to the hospital, noting that 


\section{F. P. Hopp et al.}

"I'd just make it. I know I'd need to go somewhere", while the caregiver indicated that they would advise their loved one of the need to go to the hospital, noting that "she would have no choice-she'd have to". In another dyad pair, the individual with heart failure indicated that she would go to the hospital in the given scenario, but her caregiver noted "I would convince her and keep asking over and over. Eventually, she always will say 'ok' and she'll go."

Complementary communication style. A third style, which we describe as "complementary" is one in which the caregiver and patient have similar views concerning the need to go to the hospital, and no mention is made of the need to persuade. Two of the dyads displayed this pattern. In one instance, the patient noted that "you staying at home, you're not a doctor. At the house, you ain't got nothing but your own room", while his stepson felt he would "...need to go somewhere where there are professional healthcare providers that can give him the proper care he needs." In another dyad, the individual with heart failure indicated that he would go to the hospital in the given scenario, and his caregiver agreed, noting that "He's not ready to go yet, so he would do anything and everything that he has to do to prolong his life to make sure he's still here."

Conditional communication style. A fourth style, which was present in two of the pairs, is what we term "conditional: the expected course of action would depend on the particular circumstances. One of these caregivers indicated that "if she's in her right state of mind, I would do whatever she wants to do", while another put it this way:

She'll go until she'll say, “Ok, I'm tired." Then she won't go. So if she was to say to me, "I don't want to go. I'm tired. I'm just tired." I won't make her. If the doctor told me there was nothing else that they could do and the treatment, like you said, was not working and not benefiting her, then I would not make her go.

Directive communication style. The fifth and final style, present in one of the dyads, was what we describe as a "directive" style, one in which the patient and caregiver have different views about the best course of action in a given scenario, and the caregiver indicates a perceived need to carry out their desired course of action without consideration of the patient's preferences. In this dyad, the caregiver's desire for the patient to go to the hospital was in direct contradiction with the loved one's wish to stay at home. This patient indicated that she preferred to stay at home because "if 
Decisions in Heart Failure

they [home care staff] come like they been coming, it would be about the same", while her son related that:

She told me after she came home, "If I fall on the floor, let me lay there. No, I'm not. I don't want to go to the hospital. I don't care what you want. I'm going to do what's best for you.

In this dyad, the son correctly interpreted the desire on the part of his mother to stay at home, but noted an intention to pursue what he believed to be the best course of action.

\section{Discussion}

Response to the first research question concerning expectations about going to the hospital versus staying at home when faced with deteriorating symptoms, indicated that most participants would choose to go to the hospital. These results are consistent with previous research involving both African-American and white patients with chronic disease, which found that hospitalization was often the desired outcome, especially when hospitalization was depicted as helping individuals to improve their health (Fried, 2000). However, such preferences are likely to be particularly salient for this African-American sample, given discrimination and difficulties accessing needed services experienced previously. African Americans with heart failure may choose to utilize hospital services as a way to self-monitor, access medical authorities, and avoid death despite the challenges associated with hospitalization. Moreover, for African American heart failure patients in low-income urban areas, the hospital may provide more benefits than costs when compared to other types of care.

Responses to the third research question, dealing with communication styles between persons with heart failure and their caregivers as they discussed hospital decision making, revealed a diversity of decision-making styles. A previous exploratory study found that African Americans were more likely than Caucasians to prefer a family-centered approach to discussions of advanced care planning, one that involved the active involvement of family members in making future health care decisions (Waters, 2000). The communication styles in the present study reflect this importance of family involvement in decision making. For example, two of the dyad communications styles (influential and complimentary/influential) involved an expectation that the 


\section{F. P. Hopp et al.}

caregiver would play a role in convincing or persuading their loved one to go to the hospital. This suggests that, although there is broad agreement on the need for hospitalization, caregivers perceived that, at the actual time to decide, their loved one will express a reluctance to go and would require persuasion. The directive style is particularly important to consider because it suggests that, in some instances, patient autonomy may not be a component of the decision-making style and that instead, the desire of the caregiver may take precedence over the wishes of the person with heart failure. The potential presence of a directive style highlights the ethical obligation of social workers and other key members of the treatment team to ensure that the wishes of persons with heart failure are honored (Munthe, Sandman, \& Cutas, 2012). Recognizing the diversity in communication styles is important, as it highlights and supports the importance of heterogeneity within the African American community, and the need to move towards a multidimensional, dynamic approach to diversity that recognizes a diversity of communication styles that can impact communication around health-care issues (Celious \& Oyserman, 2001; Epner \& Baile, 2012).

\section{Implications for Social Work Practice}

The present research suggests several important areas of focus for social workers who work with heart failure patients. The most important contribution that social workers can make is to advocate for greater recognition of the diverse ways in which communication patterns are formed and expressed across and within different racial and cultural groups. Social work values emphasize the promotion of social justice and the reduction of inequalities, suggesting that the profession needs to develop culturally competent interventions for diverse populations, especially when different racial groups have disparities in health conditions and outcomes, as is the case among African Americans experiencing heart failure. Social workers can expand knowledge of cultural experiences and perceptions, recognize and discuss the heterogeneity within the African-American population, and collaborate in the development of more effective and culturally appropriate services, in both the home and the hospital.

Social workers can also advocate for and support persons with heart failure who face difficult care decisions. This study suggests that many African Americans with heart failure are interested in keeping track of 
symptoms and seeking hospitalization to understand those symptoms. Social workers should be aware of this desire and promote and advocate for ways that older adults can access self-care and self-monitoring at home. Since study participants anticipate experiencing challenges during hospitalization, and they may perceive a lack of availability of there is a need for social workers to advocate for policies to support adequate hospital and community-based services for these persons.

A related role for social workers is to recognize and support different expectations about hospitalization, as well as variation in communication styles that exist between people with heart failure and their caregivers. Social workers can play an important role in recognizing the different ways that persons with heart failure make decisions, including the different decision-making styles identified in the present study (influential, collaborative-influential, complimentary, conditional, and directive). Moreover, although most of the pairs (9 of 11) expressed similar views about their expected decision, two have different perceptions. Specifically, one of the persons with heart failure indicated that she would want to go to the hospital, but her caregiver expected that she would want to stay at home, In another, the individual with heart failure indicated that she would want to stay at home, while the caregiver thought she would want to go to the hospital. These responses suggest the need for social workers to facilitate greater communication between persons with heart failure and their caregivers, recognizing the potential for discrepancies in perceptions as well as the presence of differing communication styles. Social workers can help inter-disciplinary health-care teams to understand the need to recognize and address these communication issues and associated challenges. Particularly in instances where a directive communication style is present, social workers can help ensure that the views and wishes of both patients and caregivers are given voice. They can also play a critical role in facilitating more frequent and in-depth discussions between patients and caregivers concerning medical treatment decisions, so that families can better communicate and work together.

\section{Areas for Future Research}

This study suggests three major areas for future research. First, studies are needed to obtain information from recently hospitalized older persons and their family members on hospitalization decisions. Quite possibly, a different pattern of communication styles will become evident when 


\section{F. P. Hopp et al.}

respondents are faced with an actual decision, rather than responding to a hypothetical. Second, findings indicating the perception of many caregivers that they will need to persuade their loved one to go to the hospital or to use a directive approach suggests the need to develop and test interventions that provide those with heart failure and their family members the chance to talk about preferences for hospitalization in advance. Such conversations are likely to clarify preferences and expectations for medical care and ensure that those with heart failure are able to access care that is consistent with their preferences and goals. Third, there is a need for more information concerning communication styles around hospitalization decisions among different racial and ethnic groups, since this study is among the first to identify varying styles within the African American community.

\section{Study Limitations}

Three major limitations to our study should be noted. First, the study is based on a small but purposive sample ( $\mathrm{n}=11$ dyads) from a larger qualitative study. Although the overall sample for this study is small, it should be noted that especially for homogenous groups, variability of code definitions can be stable after only a few interviews (Guest, Bunce, \& Johnson, 2006). This indicates that important themes can still be gleaned from qualitative studies based on a small sample size. However, future studies should be conducted to determine whether the current findings are transferable to other health care settings and cultural contexts. Only African Americans with heart failure who gave permission for their caregiver(s) to be interviewed were included in this secondary analysis. Those who did not have a caregiver, or did not wish to nominate a caregiver, were not included. Since the only caregivers included in the sample were those who had loved ones with heart failure who were willing to nominate them for possible participation, the sample likely represented a select group of individuals who had particularly close relationships with their caregivers. Although the small sample size is consistent with the goal of qualitative research to provide in-depth information, the results are not generalizable to the larger population of persons with heart failure and their caregivers. Moreover, the results are not likely to reflect the experiences of dyads with less intimate relationships. Therefore, future studies should be based on larger samples and be designed to expand knowledge of decision making among caregivers who have more distant and/or conflicted relationships. 
Decisions in Heart Failure

Second, inferences about communication style were derived from separate interviews among persons with heart failure and their caregivers. We did not have data on discussions that took place between patients and their caregivers on their preferences for hospitalization in the illness scenario. To better discern the nature of the communication style between persons with heart failure and their caregivers information on such discussions should be considered for future research.

A third and final limitation is that we reviewed medical records for evidence of dementia-related illness, but did not directly assess decisionmaking capacity of the persons with heart failure who participated in the study. A more thorough investigation of the capacity of potential participants to engage in discussions about hospitalization decisions would be helpful for future studies. A recent study of persons with mild to moderate dementia and their caregivers suggests that persons with this level of dementia often contribute to research participation decisions and have the ability to do so (Black, Wechsler M \& Fogarty, 2013). Quite possibly this capacity could extend to their ability to participate in decisions about hospitalization.

\section{Conclusions}

This research points to the need for additional inquiries into the ways people with heart failure and their caregivers make hospitalization decisions. Such information is critical for social workers who work in an environment characterized by increasing concern about the high costs associated with hospital care, particularly at the end of life (Hogan, Lunney, Gabel, \& Lynn, 2001; Riley \& Lubitz, 2010). With health-care environments increasingly focused on containing hospitalization costs, social workers have a particular obligation to advocate for African Americans with heart failure, by promoting comprehensive services and care settings that are consistent with the expressed needs of these persons and others with chronic diseases. (National Association of Social Workers, 2001).

\section{Acknowledgements}

This study was funded by the John A. Hartford Foundation Geriatric Social Work Faculty Scholars Program, for the project entitled Barriers and Facilitators to Heart Failure Care in Urban Areas (Faith Hopp, Principal 
F. P. Hopp et al.

Investigator). We express our appreciation to Drs. Letha Chadiha and Ruth Dunkle of the University of Michigan School of Social Work, and to the members of the writing group sponsored by the NIA Training Grant (Social Research Training on Applied Issues in Aging, School of Social Work, University of Michigan), for their support and feedback on this manuscript.

\section{References}

Allen, R., Allen, J., Hilgeman, M., \& DeCoster, J. (2008). End-of-life decision-making, decisional conflict and enhanced information: Race effects. Journal of the American Geriatrics Society, 56(10), 1904-1909.

Allen JW, Arslanian-Engoren C, \& Lynch-Sauer J. (2009). The lived experience of middle-aged women with New York Heart Association class III heart failure: A pilot study. Progress in Cardiovascular Nursing, 24(3), 96-101.

Allen L.A., Stevenson, L.W., Grady, K.L, Goldstein, N.E., Matlock, D.D., Arnold, R.M...Spertus, J.A. (2012). Decision making in advanced heart failure: A scientific statement from the American Heart Association. Circulation, 125, 1928-1952.

American Heart Association (2013). African Americans \& cardiovascular diseases: Statistical fact sheet 2013 update. Retrieved from http://www.heart.org/idc/groups/heart-

public/@wcm/@sop/@smd/documents/downloadable/ucm_31956 8.pdf.

Black, B.S., Wechsler M, \& Fogarty L. (2013). Decision making for participation in dementia research. American Journal of Geriatric Psychiatry 21(4):355-63.

Braun, V. \& Clarke, V (2006). Using thematic analysis in psychology. Qualitative Research in Psychology, 3, 77-101.

Braun, U., Beyth, R., Ford, M., \& McCullough, L. (2008). Voices of African American, Caucasian, and Hispanic surrogates on the burdens of end-of-life decision making. Journal of General Internal Medicine, 23, 267-274.

Brown, D.W., Haldeman, G.A., Croft, J.B., Giles, W.H. \& Mensah, G.A. (2005). Racial or ethnic differences in hospitalization for heart failure among elderly adults: Medicare, 1990 to 2000. American Heart Journal, $150,448-454$.

Cardiovascular Outcomes (2009). Retrieved from http:// cvoutcomes.org 
Decisions in Heart Failure

Celious, A. \& Oyserman, D. (2001). Race from the inside: An emerging heterogeneous race model. Journal of Social Issues, 57(1), 149-165.

Deswal, A., Petersen, N.J., Urbauer, D.I., Wright, S.M., \& Beyth, R. (2006). Racial variations in quality of care and outcomes in an ambulatory heart failure cohort. American Heart Journal, 152(2), 348- 354.

Dolgin, M. (1994). Nomenclature and criteria for diagnosis of diseases of the heart and great vessels (9 $9^{\text {th }}$ ed.). Boston, MA: Little, Brown \& Co.

Epner D.E., \& Baile, W.F. (2012). Patient-centered care: The key to cultural competence. Annals of Oncology: Official Journal of the European Society of Medical Oncology, 23(3), 33-42.

Ferguson, J.A. et al. (1998). Racial disparity in cardiac decision making: results from patient focus groups. Archives of Internal Medicine, 158 (13), 1450-1453.

Franciossa, J.A., Ferdinand, K.C., \& Yancy, C.W. (2010). Treatment of heart failure in African Americans: A consensus statement. Congestive Heart Failure, 16(1), 27-38.

Fried, T., van Doorn, C., O'Leary, J., Tinetti, M., \& Drickamer, M. (2000). Older persons' preferences for home vs. hospital care in the treatment of acute illness. Archives of Internal Medicine, 160, 1501-1506.

Freidman, B., Jiang, H.J. and Russo, C.A. (2009). Medicare Hospital Stays: Comparisons between the Fee-for-Service Plan and Alternative Plans, 2006. HCUP Statistical Brief \#66. January 2009. Agency for Healthcare Research and Quality, Rockville, MD. http://www.hcupus.ahrq.gov/reports/statbriefs/sb66.pdf

Givens, JL, Tjia, J., Zhou, C. Emanuel, E. \& Ash A. (2010). Racial and ethnic differences in hospice use among patients with heart failure. Archives of Internal Medicine, 170(5), 427-432.

Guest, G., Bunce, A., \& Johnson, L. (2006). How many interviews are enough? An experiment with data saturation and variability. Field Methods, 18(1), 59-82.

Haley, W. E., Allen, R. S., Reynolds, S., Chen, H., Burton, A., \& GallagherThompson, D. (2002). Family issues in end-of-life decision making and end-of-life care. American Behavioral Scientist, 46, 284-298.

Hebert, R.S., Schulz, R., Copeland, V.C., \& Arnold, R.M. (2009). Preparing family caregivers for death and bereavement. Insights from caregivers of terminally ill patients. Journal of Pain and Symptom Management, 37(1), 3-37.

Hobart, K. (2002). Death and dying and the Social Work role. Journal of Gerontogical Social Work, 36(3-4), 181-192. 


\section{F. P. Hopp et al.}

http://dx.doi.org/10.1300/J083v36n03_14.

Hogan, C., Lunney, J., Gabel, J., \& Lynn, J. (2001). Medicare beneficiaries' cost of care in the last year of life. Health Affairs, 20(4), 188-195.

Hopp, F.P., Thornton, N., Martin, L., \& Zalenski, R. (2011). Life disruption, life continuation: Contrasting themes in the lives of AfricanAmerican elders with advanced heart failure. Social Work in Health Care, 51(2):149-72.

Horowitz, A., Goodman, C.R., \& Reinhardt, J.P. (2004). Congruence between disabled elders and their primary caregiver. The Gerontologist, 44(4), 532- 542.

Horowitz CR, Rein SB, Leventhal H. (2004). A story of maladies, misconceptions and mishaps: Effective management of heart failure. Social Science and Medicine, 58(3), 631-643

Husaini et al. (2011). Race, sex, and age differences in heart failure-related hospitalizations in a southern state: Implications for prevention. Circulation: Heart Failure, 2, 161-9.

Joynt, K.E., Orav, E.J., \& Jha, A.K. (2011). Thirty-day readmission rates for Medicare beneficiaries by race and site of care. Journal of the American Medical Association, 305(7), 675-681.

LaVeist, T.A., Nickerson, K.J., \& Bowie, J.V. (2000). Attitudes about racism, medical mistrust, and satisfaction with care among African American and White cardiac patients. Medical Care Research and Review, 57(1), 146-161.

Lee, D.S., Austin, P.C., Rouleau, J.L., Liu, P.P., Naimark, D., \& Tu, J.V. (2003). Predicting mortality among patients hospitalized for heart failure: Derivation and validation of a clinical model. Journal of the American Medical Association, 290, 2581- 2587.

Loehr L.R., Rosamond W.D.D, Chang, P.P., Folsom A.R., Chambless L.E.(2008). Heart failure

incidence and survival (From the Atherosclerosis Risk in Communities [ARIC] study). American Journal of Cardiology, 101 (7):1016-22

Lyons, K.S., Zarit, S.H., Sayer, A.G., \& Whitlatch, C.J. (2002). Caregiving as a dyadic process: Perspectives from caregiver and receiver. Journal of Gerontology: Psychological Sciences, 57B(3), 195- 204.

Matthews, A.K., Sellergren, S.A., Manfredi, C., \& Williams, M. (2010). Factors influencing medical information seeking among African American cancer patients. Journal of Health Communication: International Perspectives, 7(3), 205-219.

Mitchell, J.E., Ferdinand, K.C., Watson, K.E., Wenger, N.K., Watkins, L.O., 
Decisions in Heart Failure

Flack, J.M.,...Wright, J.T. (2011) Treatment of heart failure in African Americans- A call to action. Journal of the National Medical Association, 103(2), 86- 98.

Munthe, C., Sandman, L., \& Cutas, D. (2012). Person centered care and shared decision making: Implications for ethics, public health and research. Health Care Analysis, 20(3), 231- 249.

Nakashima, M., Chapin, R.K., Macmillan, K., \& Zimmerman, M. (2005): Decision Making in Long-Term Care. Journal of Gerontological Social Work, 43(4), 79-102.

National Association of Social Workers (2001). NASW standards for cultural competence in social work practice. Washington, DC: National Association of Social Workers.

Padgett, D. (1998). Qualitative methods in social work research: Challenges and rewards, (2nd ed.) Sage Publications, Thousand Oaks.

Retrum, J.H., Nowels, C.T., \& Bekelman, D.B. (2012). Patient and caregiver congruence: The importance of dyads in heart failure care. Journal of Cardiovascular Nursing, February 17 [epub ahead of print]

Riley, G.F. \& Lubitz, J.D. (2010). Long-term trends in Medicare payments in the last year of life. Health Services Research, 45(2), 565- 576.

Sayers, S.L., White, T., Zubritsky, C., \& Oslin, D.W. (2006). Family involvement in the care of healthy medical outpatients. Family Practice, 23, 317- 324.

Sebern, M., \& Riegel, B. (2009). Contributions of supportive relationships to heart failure self-care. European Journal of Cardiovascular Nursing, $8(2), 97-104$.

Shin, D.W., Cho, J., Roter, D.L., Kim, S.L., Sohn, S.K., Yoon, M., ...Park, J. (2013). Preferences for and experiences of family involvement in cancer treatment decision-making: Patient-caregiver dyads study. Psycho-Oncology.

Thorne, S., Oliffe, J.L., \& Stadjuhar, K.L. (2013). Communicating shared decision-making: Cancer patient perspectives. Patient Education and Counseling, 90(3), 291-6.

Torke, A. M., Corbie-Smith, G.M, \& Branch, W.T. (2004). African American patients' perspectives on medical decision making, Archives of Internal Medicine, 164, 525-530.

Waters, C.M. (2000). End-of-life care directives among African Americans: lessons learned--a need for community-centered discussion and education. Journal of Community Health Nursing, 17(1), 25-37.

Yancy, C.W. (2005). Heart failure in African Americans. The American 


\section{F. P. Hopp et al.}

Journal of Cardiology, 96(7B), 3i- 12

Zambroski CH. (2003). Qualitative analysis of living with heart failure. Heart and Lung. 2003 32(1):32-40. 